\title{
Meta
}

Journal des traducteurs

Translators' Journal

\section{Contribution à la bibliographie lexicographique médicale en langue espagnole}

\section{Henri Van Hoof}

Volume 46, numéro 1, mars 2001

Traduction médicale et documentation / Medical translation and documentation

URI : https://id.erudit.org/iderudit/004552ar

DOI : https://doi.org/10.7202/004552ar

Aller au sommaire du numéro

Éditeur(s)

Les Presses de l'Université de Montréal

ISSN

0026-0452 (imprimé)

1492-1421 (numérique)

Découvrir la revue

Citer ce document

Van Hoof, H. (2001). Contribution à la bibliographie lexicographique médicale en langue espagnole. Meta, 46(1), 179-182. https://doi.org/10.7202/004552ar d'utilisation que vous pouvez consulter en ligne. 


\title{
Contribution à la bibliographie lexicographique médicale en langue espagnole
}

\author{
HENRI VAN HOOF \\ Institut libre M. Haps, Bruxelles, Belgique
}

MÉDECINE EN GÉNÉRAL

Cardenal, L. (1966) : Diccionario terminológico de ciencias médicas, $9^{\text {a }}$ ed., Barcelona, Salvat. $(E l)$

Carrillo Robles, A. (1974): Gran diccionario médico, Madrid, Publicaciones Controladas. $\left(\right.$ TRAD $\left.^{1}\right)(E l)$

Clasificación internacional de enfermedades (1950-1967), Geneva, Organisation mondiale de la santé (OMS)/World Health Organization (WHO), 2 vol. - (En-Fr-El-It)

Diccionario médico de bolsillo (1976), $2^{\mathrm{a}}$ ed., Barcelona, Salvat. - (El)

Dorian, A. F. (1987-1990) : Encyclopaedic Dictionary of Medicine, Amsterdam, Elsevier, 4 vol. (En-Fr-De-El-It)

Enciclopedia de medicina, higiene y seguridad del trabajo (1974), Madrid, Instituto nacional de previsión (INP). - (El)

Enciclopedia médica básica (1976), Barcelona, Nauta. - (El)

Golberg, M. (1947-1952): Chemical and Medical Dictionary, New York, McGraw-Hill. - (En-El/ El-En)

Gómez, J. (1976) : Diccionario de sintomas, Barcelona, Acervo. - (TRAD) (El)

González-Campo de Cos, J. (1956): Diccionario médico, Barcelona, Marín y Campo.

Меувоом, F. (1961): Questionarium Medicum, Amsterdam, Elsevier. - (En-Fr-De-El-It-Ne, etc.) Gran Larousse de medicina (1977), Barcelona, Noguer. - (TRAD ex Fr) ${ }^{1}$ (El)

Mommsen, H. (1976) : Diccionario médico para la familia, 5ª ed., Barcelona, Labor. - (TRAD ex $\mathrm{De})(E l)$

Newman, W. A. (s. d.) : Diccionario de ciencias médicas Dorland, s. l., s. é. - (TRAD ex En) (El) Ruiz Torres, F. (1961) : Diccionario de medicina, $2^{a}$ ed., Madrid, Alhambra. - (En-El/El-En)

- (1971): Diccionario de medicina, $2^{\text {a }}$ ed., Madrid, Alhambra. - (De-El/El-De)

Schulz, H. E. (1963-1964) : Vocabularium medicinale polyglotte, Halle, Deutsches NomenklaturArchiv. - (Lat-En-Fr-De-El-It-Ru)

Sliosberg, A. (1975) : Dictionnaire médical, $2^{\mathrm{e}}$ éd., Amsterdam, Elsevier. - (En-Fr-De-El-It)

Valero-Ribas, J. (1961): Enciclopedia de ciencias médicas, 2a ed., Barcelona, Salvat. - (El)

Van Hoof, H. (1999): Manual práctico de traducción médica. Diccionario básico de términos médicos, Granada, Comares. - (TRAD ex Fr) (En-Fr-El)

Vanhove, H. (s. d.): Glossaire de termes reliés à la médecine, la pharmacie et la chimie, Beerse (Belg.), Jansen Pharmaceutica. - (Fr-En-De-El-It-Ne-Lat)

\section{ALLERGOLOGIE}

Wilken-Jensen, K. (1966) : Lexicon allergologicum, 2nd ed., London, Pergamon Press. - (Lat-EnFr-De-El-It-Ru)

\section{ANATOMIE}

Pequeño atlas de anatomía (1978), Frankfurt am Main, Fröhlich. - (En-De-El)

Piscitelli, N. (1974) : Diccionario atlas de anatomía humana, Barcelona, Teide. - (TRAD ex It) (El)

Wordemann, H. W. (1957) : Nomina anatómica parisiensia, Utrecht, Spectrum (En-Fr-De-El-ItPo). 
180 MetA, XLVI, 1, 2001

\section{BIOLOGIE}

Haensch, G. and G. Haberkamp de Antón (1981) : Dictionary of Biology, Amsterdam, Elsevier. - (En-Fr-De-El)

\section{CANCÉROLOGIE}

Hamperl, H. et L. V. Ackermann (1969): Nomenclature illustrée des tumeurs, $2^{e}$ éd., Berlin, Springer. - (En-Fr-De-El-Ru)

SmalbraAK, J. (1957): Trophoblastic Growths, Amsterdam, Elsevier. - (En-Fr-De-El-It)

\section{CARDIOLOGIE}

Liechty, M. (1973): Glossaire de cardiologie, Mons, École d'interprètes internationaux. - (Fr-El)

\section{DENTISTERIE ET ODONTOLOGIE}

Atateu, R. M. et al. (s. d.) : Vocabulari d'odontoestomatologia i ortodoncia, Barcelona, Termcat. (Cat-Fr-En-El)

BRUnET, M. (1971): Glossaire de parodontologie, Mons, École d'interprètes internationaux. - (Fr$E l)$

\section{DERMATOLOGIE}

Pirlot, C. (1990a) : El eccema de contacto alérgico, Bruselas, Institut supérieur de traduction M. Haps. - (El-Fr)

- (1990b) : Maladies auto-immunitaires en dermatologie, Bruxelles, Centre de terminologie de Bruxelles. - (El-Fr)

DIÉTÉTIQUE

Formells, P. (s. d.): Vocabulari de dietética i nutrició, Barcelona, Termcat. - (Cat-Fr-En-El)

\section{EMBRYOLOGIE}

VinCENT, J. (1973) : Glosario de embriología, Bruselas, Institut supérieur de traduction et d'interprétation L. Cooremans. - (El-Fr)

\section{ÉPILEPSIE}

Gastaut, H. (1973): Dictionary of Epilepsy. Definitions, Geneva, Organisation mondiale de la santé (OMS)/World Health Organization (WHO). - (En-Fr-El-Ru)

\section{GÉNIE SANITAIRE}

Terminología técnica de ingenería sanitaria (1957), México, Asociación interamericana de ingenería sanitaria. - (En-Fr-El-Po)

Moya, V. J. (1952) : Nomenclatura de ingenería sanitaria, México, Rolland. - (En-El)

\section{GYNÉCOLOGIE ET OBSTÉTRIQUE}

Louros, N. C. (1964): Glossaire des termes obstétricaux et gynécologiques, Amsterdam, Elsevier. (Lat-En-Fr-De-El-It-Ru)

\section{HÉMATOLOGIE}

Franquesa, E. i I. Llucia (s. d.) : Diccionari terminológic d'hematologia, Barcelona, Termcat. (Cat-Fr-En-El)

Romei-Braconi, L. (1964) : Hematology, Amsterdam, Elsevier. - (En-Fr-De-El-It-Ru) 


\section{HYGIÈNE}

Enciclopedia de medicina, higiene y seguridad del trabajo (1974), Madrid, Instituto nacional de previsión (INP). - (El)

\section{IMMUNOLOGIE}

Herbert, W. J. y P. C. Wilkinson (1974) : Diccionario de immunología, Barcelona, Jims. - (TRAD ex En) $(E l)$

Marchand, G. (1992): Las infecciones por VIH. Terminología y comentarios, Bruselas, Institut supérieur de traduction M. Haps.

\section{MÉDECINE HOSPITALIÈRE}

Aurousseau, P. (1977): Vademecum hospitalier international, Paris, Masson. - (En-Fr-El)

\section{MÉDECINE DU TRAVAIL}

Enciclopedia de medicina, higiene y seguridad del trabajo (1974), Madrid, Instituto nacional de previsión (INP). - (El)

\section{MÉDECINE TROPICALE}

Catherine, M. H. (1972): Glossaire de médecine tropicale, Mons, École d'interprètes internationaux. - (El-Fr)

Terminología del paludismo y de la eradicación del paludismo (1974), Geneva, Organisation mondiale de la santé (OMS)/World Health Organization (WHO). - (En-Fr-El)

\section{MYOLOGIE}

Denis, F. (1974): Terminología bilingue de miología, Bruselas, Institut supérieur de traduction et d'interprétation L. Cooremans. - (El-Fr)

\section{NEUROLOGIE}

Schietecat, C. (1996): Los trastornos del lenguaje oral en el afásico adulto. Terminología, Bruselas, Institut supérieur de traduction M. Haps. - (El-Fr)

\section{NUTRITION}

Formells, P. (s. d.) : Vocabulari de dietética i nutrició, Barcelona, Termcat. - (Cat-Fr-En-El)

Rafecas, M. (s. d.) : Vocabulari de nutrició, Barcelona, Termcat. - (Cat-Fr-En-El)

\section{OPHTALMOLOGIE}

Alvaro, M. E. (1959): Lexicon ophtalmologicum, Basel, Karger. - (Lat-En-Fr-De-El-It)

\section{ORTHOPÉDIE ET OSTÉOLOGIE}

Junghanns, H. et al. (1977) : Nomenclatura columnae vertebralis, Stuttgart, Hippokrates Verlag. (En-Fr-De-El-It-Ru)

Renchon, A. (1975): Glossaire français-espagnol d'ostéologie, Bruxelles, Institut supérieur de traduction et d'interprétation L. Cooremans. - (Fr-El)

\section{OTO-RHINO-LARYNGOLOGIE}

LARrauri, A. (1971) : Dictionnaire d'oto-rhino-laryngologie en cinq langues, Paris, Maloine. - (FrEn-De-El-It) 


\section{PÉDIATRIE}

OpItz, H. (1974) : Encyclopedia pediátrica, Madrid, Morata. - (TRAD ex De) (El)

Sмiтн, A. K. (1948) : A Glossary of certain Child Welfare Terms, Washington D.C., US Children's Bureau Publications. - (En-Fr-El-Po)

\section{PHARMACOLOGIE ET PHARMACIE}

A Glossary of Psychopharmaka, A (1963), Philadelphia, Biological and Medical Services. - (EnDe- $E l)$

Kleiner Sprachführer für die pharmazeutische Praxis (1973), Stuttgart, Deutscher ApothekerVerlag. - (En-Fr-De-El-It, etc.)

Planas Guiral, F. (s. d.) : Glosario terminológico de ciencias farmaceúticas, La Habana, Ministerio de la Salud Pública. - (En-El-Fr)

Riera, C. (s. d.) : Diccionari de farmácia galénica, Barcelona, Termcat. - (Cat-Fr-En-El)

Rosenstein, E. y A. Martin del Campo (1972): Diccionario de especialidades farmaceúticas, Bogotá. - $(E l)$

Sliosberg, A. (1974-1977): Dictionary of Pharmaceutical Science and Techniques, Amsterdam, Elsevier, 2 vol. - (En-Fr-De-El-It)

Steinbichler (1963): Lexikon für die Apothekerpraxis, Frankfurt am Main, Govi. - (En-Fr-DeEl-It-Ru)

Vanhove, H. (s. d.): Glossaire de termes reliés à la médecine, la pharmacie et la chimie, Beerse (Belg.), Jansen Pharmaceutica. - (Fr-En-De-El-It-Ne-Lat)

\section{PSYCHIATRIE}

PoInso, Y. (1976) : Diccionario práctico de psicopatología, Barcelona, Herder. - (TRAD ex Fr) (El)

\section{RADIOLOGIE}

Termini radiologici (1959), München, Urban \& Schwarzenberg. - (En-Fr-De-El)

SANTÉ PUBLIQUE (cf. aussi HYGIÈNE)

Deblock, N. J. (1976) : Dictionary of Public Health, Amsterdam, Elsevier. - (En-Fr-De-El-It-Ne)

\section{TECHNIQUES MÉDICALES}

Albert, R. et H. Hahnewald (1978) : Medizintechnik, Berlin, Verlag Technik. - (En-Fr-De-El$\mathrm{Ru}$, etc.)

Wirsching, P. (1955): Katalog der medizinischen Technik, Lengdorf, Seibt. - (En-Fr-De-El)

\section{TOXICOMANIE}

Ortuña, M. (s. d.): Vocabulari de toxicomania, Barcelona, Termcat. - (Cat-Fr-En-El)

NOTE

1. L'indication TRAD signifie qu'il s'agit d'un ouvrage traduit, qui doit par conséquent exister aussi dans l'autre langue. 